\title{
Energy extremum principle for charged black holes
}

\author{
Scott Frasen ${ }^{*}$ and Shaker Von Price Funkhousen \\ Department of Physics, California Polytechnic State University, San Luis Obispo, California 93407, USA
}

\begin{abstract}
For a set of $N$ asymptotically flat black holes with arbitrary charges and masses, all initially at rest and well separated, we prove the following extremum principle: the extremal charge configuration $\left(\left|q_{i}\right|=m_{i}\right.$ for each black hole) can be derived by extremizing the total energy, for variations of the black hole apparent horizon areas, at fixed charges and fixed Euclidean separations. We prove this result through second order in an expansion in the inverse separations. If all charges have the same sign, this result is a variational principle that reinterprets the static equilibrium of the MajumdarPapapetrou-Hartle-Hawking solution as an extremum of total energy, rather than as a balance of forces; this result augments a list of related variational principles for other static black holes, and is consistent with the independently known Bogomol'nyi-Prasad-Sommerfield (BPS) energy minimum.
\end{abstract}

PACS numbers: 04.70.Bw, 04.40.Nr

\section{INTRODUCTION}

A system of multiple black holes is not static in general, but the system can be kept static by a strut (a conical singularity) on the axis between each pair of black holes. Each strut is interpreted as providing a force that prevents the black holes from moving (see [1] and references therein). Remarkably, without any struts present, there is also a well-known solution [2] that describes a set of $N$ charged black holes in static equilibrium, regardless of their mutual separations. A key feature of this static solution is that the black holes' individual charges $q_{i}$ all have the same sign, and are related to their individual masses $m_{i}$ by the condition (in units with $G=c=4 \pi \epsilon_{0}=1$ )

$$
\left|q_{i}\right|=m_{i}, \quad 1 \leq i \leq N .
$$

The asymptotically flat static geometry satisfying (1) was found by Hartle and Hawking [2, who extended the Majumdar-Papapetrou solutions [3, 4. The interpretation and significance of the condition (1) is of central interest in this paper. The traditional interpretation of the Hartle-Hawking solution 2] appeals to forces: the static equilibrium is attributed to the exact balance between the black holes' pairwise gravitational attraction and electric repulsion. However, the condition (1) refers to each black hole, not to a balance of forces between black holes. Thus, (1) is a stronger condition than a Newtonian balance of forces, which for two particles requires $q_{1} q_{2}=m_{1} m_{2}$. This suggests that (1) can be interpreted without using forces.

Such an alternative interpretation is the purpose of this paper. We show that the condition (1) can be derived from an extremum of the system's total energy $E$. This fits naturally into the framework of general relativity, where gravity and total energy are determined by

\footnotetext{
* Email address: scfraser@calpoly.edu

† Email address: sfunkhou@calpoly.edu
}

spacetime geometry, and gravity is fundamentally not treated as a force.

The condition (1) describes extremal charge, since a nonrotating black hole satisfies a charge-mass inequality, $|q| \leq m$. Thus, our results can be briefly summarized as: a set of extremally charged black holes extremizes the total energy. More precisely, we consider a conformally flat spatial geometry in the form introduced by Brill and Lindquist [5], and we prove the following extremum principle for well-separated black holes:

For $N$ charged black holes, initially at rest, each with $\left|q_{i}\right| \leq m_{i}$ and apparent horizon area $A_{i}$, the extremal charge condition $\left|q_{i}\right|=m_{i}$ follows from extremizing the total energy: $\partial E / \partial A_{i}=0$ at fixed charges $q_{i}$ and Euclidean separations $r_{i j}$.

We prove the extremum principle (2) as an expansion in the inverse separation distances $\left(1 / r_{i j}\right)$, through second order, which is where relativistic post-Newtonian contributions first appear. The particular quantities that are varied or held fixed in this energy extremum are motivated by the first law of black hole mechanics, as we will illustrate when we prove (2).

If all of the black hole charges in (2) have the same sign, then (2) is a variational principle for static black holes: it identifies a static black hole configuration as an extremum of total energy, within a family of black holes that are initially at rest. That is, a configuration with extremal energy remains at rest, while all other configurations evolve dynamically. Such variational principles have been proved for a single uncharged black hole [6], a single black hole in Einstein-Yang-Mills theory [7, and pairs of mirror-symmetric black holes in the RandallSundrum braneworld models [8]. Our extremum principle (2) adds the case of charged black holes to these earlier variational principles [6 8 ] and identifies the static Hartle-Hawking solution as its energy extremum. In contrast to [6] [ , in this paper, the number $N$ of black holes that we consider is arbitrary, and our energy extremum varies (rather than holds fixed) the black hole areas. 
We also show that the energy extremum in (2) is an energy minimum. This result is consistent with the earlier result of Gibbons and Hull [9], but our methods are different. In [9], it was found that the Majumdar-Papapetrou solution saturates the lower bound of a supersymmetric Bogomol'nyi-Prasad-Sommerfield (BPS) inequality, $E \geq|Q|$, for global quantities: the total energy $E$ and charge $Q$ (with zero magnetic charge in the context of this paper). In contrast to [9], in this paper, we minimize the energy $E\left(A_{i}, q_{i}, r_{i j}\right)$ as a function of several variables, and we do not use supersymmetry; we also obtain additional extremum conditions $\left(m_{i}=\left|q_{i}\right|\right)$ on the individual masses and charges.

It is worth noting that, although black holes with extremal charge are not often considered in astrophysical applications, they have attracted significant theoretical interest. For example, they represent stable ground states for black holes in supersymmetric theories [10] and they have been used to investigate the nature of entropy in black hole thermodynamics [11.

This paper is organized as follows. In Sec. II] we review the necessary geometry. In Sec. III], we prove the extremum principle (2) for well-separated black holes, through second order, and we specify the physical conditions under which the separations $r_{i j}$ are sufficiently large. In Sec. IV], we show that the energy extremum is a minimum, and verify that it agrees with the BPS bound. We conclude in Sec. V] Throughout this paper, we work in four spacetime dimensions and use geometric units with $G=c=4 \pi \epsilon_{0}=1$.

Much of our analysis does not require that the black hole charges have the same sign. We only need to refer to same-sign charges in the following contexts: in a higherorder analysis (Sec. IIIE), in comparisons to the BPS bound (Sec. IV), and in applications of the extremum principle (2) as a variational principle that reproduces the static Hartle-Hawking solution (as described above).

\section{GEOMETRY}

In this section, we review the geometry $[2,5$, that we use in this paper. A system of $N$ charged black holes, all initially at rest, is described by its instantaneous spatial geometry. The appropriate area $A_{i}$ of a black hole is that of its apparent horizon, which is determined by the spatial geometry alone (unlike the event horizon, which is a global spacetime property). The apparent horizon generally lies inside the event horizon, and coincides with it for a static or stationary spacetime.

We use a conformally flat geometry, in the form introduced by Brill and Lindquist [5]. This geometry contains no conical singularities (struts) to prevent the black holes from moving. The extremal black holes considered by Hartle and Hawking [2] remain eternally static. The nonextremal black holes considered by Brill and Lindquist [5] are initially at rest, and evolve dynamically thereafter [12, 13]. For both cases, the instantaneous

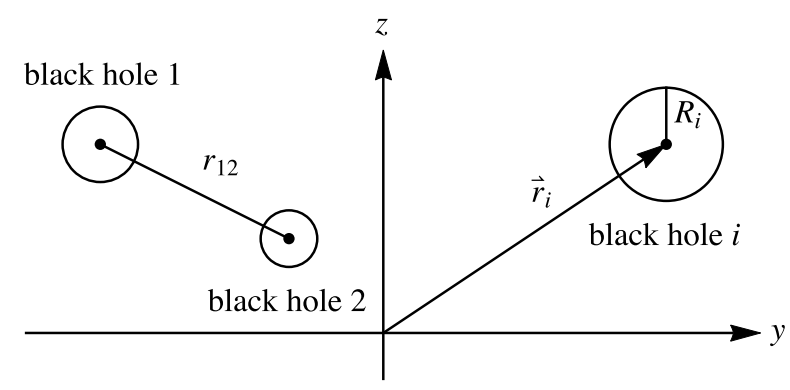

FIG. 1. Illustration of three nonextremal black holes in the spherical approximation. Black holes 1 and 2 are separated by distance $r_{12}$. Black hole $i$ has position $\vec{r}_{i}$ and apparent horizon radius $R_{i}$. Dots denote the punctures described in the text.

spatial geometry, exterior to all $N$ black holes, is

$$
\begin{aligned}
d s^{2} & =f^{2}\left(d x^{2}+d y^{2}+d z^{2}\right), \\
f & =\left(1+\sum_{i=1}^{N} \frac{\alpha_{i}}{\left|\vec{r}-\vec{r}_{i}\right|}\right)\left(1+\sum_{i=1}^{N} \frac{\beta_{i}}{\left|\vec{r}-\vec{r}_{i}\right|}\right) .
\end{aligned}
$$

Figure 1 illustrates the setup. The Euclidean vector $\vec{r}$ locates any point outside the black holes. The vector $\vec{r}_{i}$ locates black hole $i$. We let $r_{i j}$ denote a Euclidean separation distance between black holes $i$ and $j$,

$$
r_{i j}=\left|\vec{r}_{i}-\vec{r}_{j}\right| \text {. }
$$

The parameters $\alpha_{i}$ and $\beta_{i}$ are non-negative constants, and are related to physical quantities (mass, charge, energy) as follows [2, 5. Black hole $i$ has individual mass (rest energy) $m_{i}$ and charge $q_{i}$,

$$
\begin{aligned}
m_{i} & =\alpha_{i}+\beta_{i}+\sum_{j \neq i} \frac{\left(\alpha_{i} \beta_{j}+\alpha_{j} \beta_{i}\right)}{r_{i j}}, \\
q_{i} & =\beta_{i}-\alpha_{i}+\sum_{j \neq i} \frac{\left(\beta_{i} \alpha_{j}-\beta_{j} \alpha_{i}\right)}{r_{i j}} .
\end{aligned}
$$

Each pair $\left(m_{i}, q_{i}\right)$ satisfies the black hole charge-mass inequality, $\left|q_{i}\right| \leq m_{i}$. The system of $N$ black holes has total energy $E$ and interaction energy $E_{\text {int }}$,

$$
\begin{aligned}
E & =\sum_{i=1}^{N}\left(\alpha_{i}+\beta_{i}\right), \\
E_{\mathrm{int}} & =E-\sum_{i=1}^{N} m_{i}=-\sum_{i=1}^{N} \sum_{j \neq i} \frac{\left(\alpha_{i} \beta_{j}+\alpha_{j} \beta_{i}\right)}{r_{i j}} .
\end{aligned}
$$

The expressions for $m_{i}$ and $q_{i}$, in (5) and (6), can be solved for the parameters $\alpha_{i}$ and $\beta_{i}$. For large $r_{i j}$, the results to first order in $1 / r_{i j}$ are [5]

$$
\begin{aligned}
& \alpha_{i} \simeq \frac{\left(m_{i}-q_{i}\right)}{2}\left[1-\frac{1}{2} \sum_{j \neq i} \frac{\left(m_{j}+q_{j}\right)}{r_{i j}}\right], \\
& \beta_{i} \simeq \frac{\left(m_{i}+q_{i}\right)}{2}\left[1-\frac{1}{2} \sum_{j \neq i} \frac{\left(m_{j}-q_{j}\right)}{r_{i j}}\right] .
\end{aligned}
$$


Using (9), for large $r_{i j}$, the interaction energy (8) can be written to first order in $1 / r_{i j}$ as [5]

$$
E_{\mathrm{int}} \simeq-\sum_{i=1}^{N} \sum_{j>i} \frac{\left(m_{i} m_{j}-q_{i} q_{j}\right)}{r_{i j}} .
$$

This has the expected form: it is the sum of the pairwise gravitational and electrostatic potential energies.

For the static Hartle-Hawking solution [2, only one set of the parameters $\alpha_{i}$ and $\beta_{i}$ are nonzero,

$$
\left(\alpha_{i}=0, \beta_{i}>0\right) \quad \text { or } \quad\left(\beta_{i}=0, \alpha_{i}>0\right) .
$$

In terms of mass and charge, the two cases in (11) are equivalent to, respectively [2]

$$
q_{i}=m_{i}=\beta_{i}>0 \quad \text { or } \quad-q_{i}=m_{i}=\alpha_{i}>0 .
$$

Thus, the static condition, (11) and (12), can be summarized as an extremal condition for same-sign charges,

$$
\left|q_{i}\right|=m_{i}, \quad \text { with all } q_{i} \text { of like sign. }
$$

The black hole charges are nonextremal, if the parameters $\alpha_{i}$ and $\beta_{i}$ do not satisfy the static condition (11)-13. Near a nonextremal black hole $i$, we may transform from Cartesian coordinates $(x, y, z)$ in $(3)$ to spherical coordinates $(r, \theta, \phi)$ centered at $\vec{r}_{i}$. As found in [5], for sufficiently large separations $r_{i j}$, the apparent horizon of black hole $i$ can be treated as a sphere, $r(\theta, \phi)=R_{i}=$ constant. We will refer to this as the spherical approximation, which is illustrated in Fig. 1. The radius $R_{i}$ is given by [5]

$$
R_{i}{ }^{2}=\alpha_{i} \beta_{i}\left(1+\sum_{j \neq i} \frac{\alpha_{j}}{r_{i j}}\right)^{-1}\left(1+\sum_{j \neq i} \frac{\beta_{j}}{r_{i j}}\right)^{-1} .
$$

In the spherical approximation, the metric function $f$ in (3) evaluated on the surface $r=R_{i}$ is [5]

$$
f=\left(1+\frac{\alpha_{i}}{R_{i}}+\sum_{j \neq i} \frac{\alpha_{j}}{r_{i j}}\right)\left(1+\frac{\beta_{i}}{R_{i}}+\sum_{j \neq i} \frac{\beta_{j}}{r_{i j}}\right) .
$$

As we see in Sec. III the results (14) and 15 will only hold to up to an appropriate order, when expanded in powers of the inverse separations $\left(1 / r_{i j}\right)$.

We end this subsection by reviewing some technical features [2, 5] of the geometry and topology, for both nonextremal black holes and extremal black holes. The spatial geometry has the topology of $\mathbb{R}^{3}$ with $N$ points ('punctures') removed. Each puncture is located by the vector $\vec{r}_{i}$. In the flat background metric, the distance between two punctures is the Euclidean distance $r_{i j}$.

In the full conformal geometry, for a nonextremal black hole, each puncture represents the spatial infinity of an asymptotically flat region ('sheet'), which is hidden behind the black hole's apparent horizon, as viewed in the common asymptotic region (sheet) exterior to all $N$ black holes, where the total energy $E$ is defined. The topology referred to above is equivalent to the topology of $N+1$ connected sheets. Each black hole's individual mass $m_{i}$ is the Arnowitt-Deser-Misner (ADM) mass of its hidden asymptotic region (its individual sheet).

In contrast, for an extremal black hole, a careful coordinate analysis 2] reveals that the puncture at $\vec{r}_{i}$ represents the apparent horizon, with finite area. In this case, the parameter values (11) cannot be used to evaluate the apparent horizon radius (14). As shown in [2, for extremal charge, $\left|\vec{r}-\vec{r}_{i}\right| \rightarrow 0$ is a simple coordinate singularity, and the area of the apparent horizon in this limit is nonzero, $A_{i}=4 \pi m_{i}{ }^{2}=4 \pi q_{i}{ }^{2}$. This coordinate issue will pose no complications in our proof of the extremum principle in Sec. IIIB, where we will perform all calculations in the nonextremal regime, and then express the energy $E$ as function of the finite areas $A_{i}$. These areas reproduce the correct values $A_{i}=4 \pi m_{i}{ }^{2}=4 \pi q_{i}{ }^{2}$ when we take the extremal limit in terms of charges and masses $\left(\left|q_{i}\right| \rightarrow m_{i}\right)$, which is a coordinate-independent limit.

\section{ENERGY EXTREMUM PRINCIPLE}

\section{A. Physical motivation}

In this subsection, we illustrate how the first law of black hole mechanics motivates our extremum principle (2). This also demonstrates the basic extremum procedure that we use in our proof of the extremum principle (Secs. IIIB III E).

For clarity, we begin with the case of a single black hole, and then generalize it to the multi-black hole case. A single static charged black hole (the ReissnerNordström solution) has energy $E=m$ and charge $|q| \leq m$. Variations conserve energy via the first law of black hole mechanics 14,

$$
\delta E=\frac{\kappa}{8 \pi} \delta A+\Phi \delta q .
$$

Here $A, \kappa$, and $\Phi$ are the black hole's surface area, surface gravity, and electric potential, respectively. In the case of extremal charge $(|q|=m)$, it is well known that $\kappa=0$, so the first law reduces to

$$
\delta E=\Phi \delta q .
$$

The key point of (17) is the following: if $|q|=m$, then variations that hold $q$ constant $(\delta q=0)$ extremize the energy $(\delta E=0)$, and this means $\partial E / \partial A=0$, since $E$ is a function of $A$ and $q$. Conversely, if $q$ is held constant in (16), then an energy extremum requires $\partial E / \partial A=0$, and this reduces to $|q|=m$, as follows. The total energy of the Reissner-Nordström solution is

$$
E=\sqrt{\frac{\pi}{A}}\left(\frac{A}{4 \pi}+q^{2}\right) .
$$


Evaluating $\partial E / \partial A=0$ for 18 yields $|q|=\sqrt{A / 4 \pi}$, for which $E=m=\sqrt{A / 4 \pi}=|q|$ and hence $|q|=m$. In summary, the extremum $\partial E / \partial A=0$ occurs if and only if the extremal charge property holds, $|q|=m$.

As already noted, the vanishing coefficient of $\delta A$ in the first law (17) motivates why, for an arbitrarily charged black hole, we extremize the energy $(18)$ by varying the area $A$ and holding the charge $q$ constant. This generalizes to the multi-black hole case, as follows. The expected first law for the static Hartle-Hawking solution [2] is

$$
\delta E=\sum_{i=1}^{N} \Phi_{i} \delta q_{i}
$$

where $E$ is the total energy. For arbitrarily charged black holes, in analogy with the single-black hole case, we extremize $E$ by varying the black hole areas $A_{i}$ while holding constant all other quantities: the charges $q_{i}$ (which appear in the first law) and also the black hole separations $r_{i j}$ (which are internal parameters of the geometry). This motivates our extremum principle (2).

Note that the single black hole considered above remains at rest, so it illustrates our energy extremum procedure, but not any subsequent dynamics. In this paper, the initially static multi-black hole configurations that are nonextremal will not remain static; their dynamics were studied in 12 .

\section{B. Outline of proof}

In Secs. IIIC IIIE below, we will prove the extremum principle (2) as an expansion in the inverse separations, $1 / r_{i j}$. Zeroth order $\left(1 / r_{i j} \rightarrow 0\right)$ corresponds to $r_{i j} \rightarrow \infty$, and treats each black hole in isolation from the other black holes. Through first order, we retain all terms linear in $1 / r_{i j}$, which characterize effectively Newtonian interactions among the black holes. Through second order, we retain all terms quadratic in $1 / r_{i j}$, which characterize post-Newtonian relativistic interactions.

All of the terms in our expansions will be dimensionless ratios of black hole properties $\left(\alpha_{i}, \beta_{i}, m_{i}, q_{i}\right)$ divided by the separations $r_{i j}$. These ratios are small for sufficiently large separations. In particular, we will show that the extremum principle holds for sufficiently well separated black holes, as specified by conditions on $r_{i j}$ that take the form $U_{i}>0$, where $U_{i}$ can be interpreted as the effective gravitational potential experienced by black hole $i$.

At each order, the main steps are to calculate each black hole's apparent horizon area $A_{i}$, and then to obtain the energy $E\left(A_{i}, q_{i}, r_{i j}\right)$ as a function of the areas, charges, and separations. We then extremize the energy as $\partial E / \partial A_{i}=0$, and from this obtain the condition $\left|q_{i}\right|=m_{i}$, which proves the extremum principle.

\section{Proof through first order}

We begin by calculating the apparent horizon area $A_{i}$ of each black hole. As in the original work of Brill and Lindquist [5], through first order in $1 / r_{i j}$, we use the spherical approximation (see Sec. II), for which the apparent horizon is the surface $r=R_{i}=$ constant in spherical coordinates $(r, \theta, \phi)$ centered at $\vec{r}_{i}$. Then the differential area is

$$
d A_{i}=f^{2} R_{i}{ }^{2} \sin ^{2} \theta d \theta d \phi,
$$

where $R_{i}$ and $f$ are given by (14) and (15), respectively. Integrating then yields the area formula:

$$
A_{i}=4 \pi R_{i}{ }^{2} f^{2} .
$$

We now evaluate this using (14) and (15). Expanding through first order in $1 / r_{i j}$ gives

$$
\sqrt{\frac{A_{i}}{4 \pi}}=m_{i}+\sqrt{\alpha_{i} \beta_{i}}\left(2+\sum_{j \neq i} \frac{\alpha_{j}+\beta_{j}}{r_{i j}}\right),
$$

with $m_{i}$ given by (5) as a function of the other parameters $\left(\alpha_{i}, \beta_{i}, r_{i j}\right)$. We can express the area $A_{i}$ completely in terms of the mass $m_{i}$ and charge $q_{i}$, by substituting $\alpha_{i}$ and $\beta_{i}$ in (9). Then simplifying yields

$$
\begin{aligned}
\sqrt{\frac{A_{i}}{4 \pi}}= & m_{i}+\sqrt{m_{i}{ }^{2}-q_{i}{ }^{2}} \\
& \times\left(1-\sum_{j \neq i} \frac{m_{j}}{2 r_{i j}}\right)\left(1+\sum_{j \neq i} \frac{m_{j}}{2 r_{i j}}\right) .
\end{aligned}
$$

When we expand this expression, the terms that are first order in $1 / r_{i j}$ exactly cancel. At first order, we neglect the terms quadratic in $1 / r_{i j}$, and obtain

$$
\sqrt{\frac{A_{i}}{4 \pi}}=m_{i}+\sqrt{m_{i}^{2}-q_{i}{ }^{2}} .
$$

Solving this for the mass $m_{i}$ yields

$$
m_{i}=\sqrt{\frac{\pi}{A_{i}}}\left(\frac{A_{i}}{4 \pi}+q_{i}{ }^{2}\right) .
$$

Due to the cancellation that occurred to obtain (24), the mass (25) contains neither first-order terms (proportional to $1 / r_{i j}$ ) nor any parameters related to the other black holes $(j \neq i)$. This first-order result is therefore the same as the zeroth-order result, and it has the same form as the energy (18) for a single black hole.

We now evaluate the total energy $E$, which by (8) and (10) is, to first order in $1 / r_{i j}$,

$$
E=\sum_{i=1}^{N} m_{i}-\sum_{i=1}^{N} \sum_{j>i} \frac{\left(m_{i} m_{j}-q_{i} q_{j}\right)}{r_{i j}} .
$$


The zeroth-order term in 26) is the sum of the rest energies, and the first-order terms are the pairwise gravitational and electrostatic potential energies; this will be useful in interpreting the quantity $U_{i}$ below.

We now take each mass $m_{i}$ in 26 to be given by (25), which expresses the energy (26) in the functional form $E\left(A_{i}, q_{i}, r_{i j}\right)$. Then holding constant the charges $q_{i}$ and separations $r_{i j}$, we find

$$
\frac{\partial E}{\partial A_{i}}=U_{i} \frac{\partial m_{i}}{\partial A_{i}}
$$

where

$$
U_{i}=1-\sum_{j \neq i} \frac{m_{j}}{r_{i j}} .
$$

We now extremize the total energy $E$ by requiring $\partial E / \partial A_{i}=0$, which by (27) is equivalent to

$$
U_{i} \frac{\partial m_{i}}{\partial A_{i}}=0 .
$$

For sufficiently large separations, $U_{i} \neq 0$, as seen by $(28)$. In this case, the extremum 29) requires

$$
\frac{\partial m_{i}}{\partial A_{i}}=0
$$

Evaluating this for 25 reduces to

$$
\left|q_{i}\right|=\sqrt{\frac{A_{i}}{4 \pi}} .
$$

For this charge value, the mass 250 is

$$
m_{i}=\sqrt{\frac{A_{i}}{4 \pi}}=\left|q_{i}\right| \text {. }
$$

Thus, since the extremum 290 has produced the $N$ desired conditions, $\left|q_{i}\right|=m_{i}$, we have proved the extremum principle 22 through first order.

As noted above, our proof requires $U_{i} \neq 0$. As seen by (28), this condition is always satisfied at zeroth order $\left(1 / r_{i j} \rightarrow 0\right.$ and $\left.U_{i}=1\right)$, and it is satisfied at first order for sufficiently large separations $r_{i j}$ compared to the masses $m_{i}$. In particular, the continuity of our perturbative approach with the zeroth-order limit $\left(U_{i}=1\right)$ requires $0<U_{i} \leq 1$.

We end this subsection by interpreting the quantity $U_{i}$. Note that $U_{i}$ can be obtained from the energy (26) in the form $E\left(m_{i}, q_{i}, r_{i j}\right)$, along with (27) and the chain rule,

$$
U_{i}=\frac{\partial E}{\partial m_{i}} .
$$

This expression motivates the interpretation of $U_{i}$ as the effective gravitational potential experienced by black hole $i$, including its rest energy. This interpretation is justified by the derivation of (27)-(28) from (26), which makes clear that: (i) the zeroth order term in (28) is the rest energy, normalized per unit mass; (ii) the first-order sum in (28) is the Newtonian gravitational potential; (iii) there is no electric potential contribution to $U_{i}$ since 27$)-(28)$ are obtained by holding the charges constant in (26).

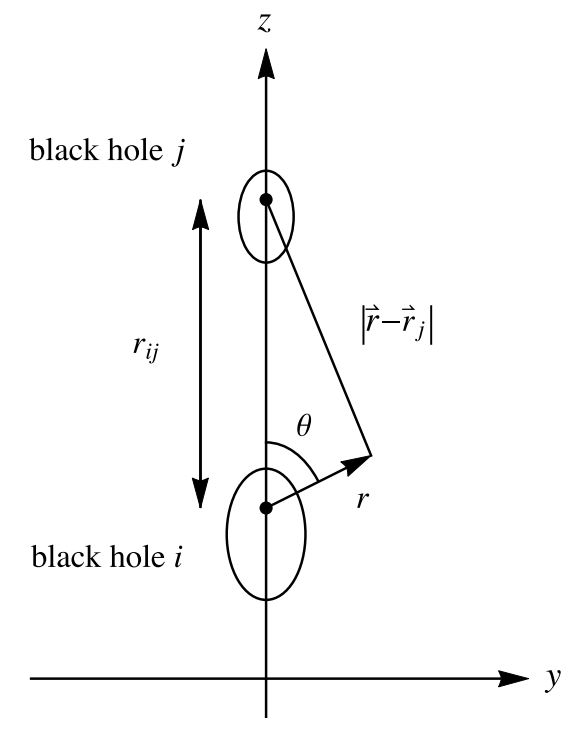

FIG. 2. Illustration of two nonextremal black holes in the second-order analysis, showing useful distances and coordinates $(r, \theta)$. The dots have Euclidean separation $r_{i j}=\left|\vec{r}_{i}-\vec{r}_{j}\right|$. All black holes (including others, not shown) are collinear on the $z$ axis, and have azimuthal symmetry about the $z$ axis.

\section{Proof through second order}

In this section, we prove the extremum principle (2) through second order. Our first task will be to evaluate each apparent horizon area $A_{i}$. This is a longer calculation than in our first-order proof (Sec. III C), since at second order, we must depart from the spherical approximation. Figure 2 illustrates the setup.

In general, each black hole's apparent horizon is nonspherical, due to its interactions with the other black holes. Thus, in spherical coordinates $(r, \theta, \phi)$ centered at $\vec{r}_{i}$, the apparent horizon of black hole $i$ is generally a surface $r(\theta, \phi)$. To simplify the analysis, we will take all black holes to be collinear (aligned on the $z$ axis). The resulting axisymmetry simplifies each nonspherical horizon $r(\theta, \phi)$ to the azimuthally symmetric form $r(\theta)$. Thus, as illustrated in Fig. 2, we take all $N$ black hole positions $\vec{r}_{i}$ to lie on the $z$ axis. Near black hole $i$, we introduce spherical coordinates $(r, \theta, \phi)$ centered at $\vec{r}_{i}$, and consider another black hole labeled by $j \neq i$. This gives

$$
\begin{aligned}
\left|\vec{r}-\vec{r}_{i}\right| & =r, \\
\left|\vec{r}-\vec{r}_{j}\right| & =\sqrt{r^{2}-2 r_{i j} \cos \theta+r_{i j}{ }^{2}} .
\end{aligned}
$$

To calculate the area $A_{i}$, we only need to consider points $(r, \theta)$ on the apparent horizon, so $r<r_{i j}$. In this case, the distance (34b) has a standard expansion in Legendre polynomials $P_{k}$,

$$
\frac{1}{\left|\vec{r}-\vec{r}_{j}\right|}=\frac{1}{r_{i j}} \sum_{k \geq 0}\left(\frac{r}{r_{i j}}\right)^{k} P_{k}(\cos \theta) .
$$


The form 35 is very suitable for our purposes, since it is an expansion in the inverse separations $\left(1 / r_{i j}\right)$. Our first-order proof (Sec. III C), like the original work of Brill and Lindquist [5], used the summation in (35) through $k=0$ and $P_{0}=1$. Here, our second-order proof proceeds through $k=1$ and $P_{1}=\cos \theta$.

We now use (34) and (35) to evaluate the metric function $f$ in (3) near black hole $i$. We retain all terms through second order in $1 / r_{i j}$. This gives

$$
\begin{aligned}
f(r, \theta)=1+ & \frac{\alpha_{i}+\beta_{i}}{r}+\frac{\alpha_{i} \beta_{i}}{r^{2}}+\sum_{j \neq i} \frac{\alpha_{j}}{r_{i j}} \sum_{k \neq i} \frac{\beta_{k}}{r_{i k}} \\
+\sum_{j \neq i} & {\left[\left(\frac{1}{r_{i j}}+\frac{r \cos \theta}{r_{i j}{ }^{2}}\right)\right.} \\
& \left.\times\left(\alpha_{j}+\beta_{j}+\frac{\alpha_{i} \beta_{j}+\beta_{i} \alpha_{j}}{r}\right)\right] .
\end{aligned}
$$

This contains all of the second-order terms from expanding the spherical approximation (15), and generalizes it by including nonspherical terms, proportional to $\cos \theta$.

Each black hole's apparent horizon $r(\theta)$ is an extremal surface, so it is determined by extremizing the area [5],

$$
A_{i}=2 \pi \int_{0}^{\pi} d \theta \sin \theta r \sqrt{r^{2}+\dot{r}^{2}} f^{2},
$$

where $\dot{r}=d r / d \theta$. Extremizing (37) gives

$$
\frac{r+\ddot{r}}{1+\dot{r}^{2} / r^{2}}=-\dot{r}\left(\cot \theta+\frac{2 \partial_{\theta} f}{f}\right)+r\left(3+\frac{2 r \partial_{r} f}{f}\right) .
$$

As in [5], we will solve (38) by expanding in Legendre polynomials $P_{n}$ as basis functions. This function-basis expansion for $r(\theta)$ is distinct from our previous multipole expansion (35). Thus, we take

$$
r(\theta)=\sum_{n \geq 0} C_{n i} P_{n}(\cos \theta),
$$

where $C_{n i}$ are constant coefficients and $i$ labels the black hole. Our first-order proof (Sec. III C), like the original work of Brill and Lindquist [5], used the expansion (39) through $n=0$, which is the spherical approximation (14). At second order, we use (39) through $n=1$,

$$
r(\theta)=C_{0 i}+C_{1 i} \cos \theta,
$$

with $P_{0}=1$ and $P_{1}=\cos \theta$. To determine the coefficients $C_{n i}$, we evaluate the differential equation 38 for $r(\theta)$ using 40 and the metric function $f$ in 36 . After expanding all quantities to second order in $1 / r_{i j}$, 38 then takes the form

$$
F_{0} P_{0}+F_{1} P_{1}(\cos \theta)=0,
$$

where $F_{0}$ and $F_{1}$ are functions (which for brevity we do not list here) that are independent of $\theta$, but involve the other parameters $\left(\alpha_{i}, \beta_{i}, r_{i j}\right)$. Since (41) is an expansion in the basis functions $P_{n}$, both $F_{0}$ and $F_{1}$ must vanish. Solving the equation $F_{0}=0$ reduces to

$$
C_{0 i}=R_{i},
$$

where $R_{i}$ is the radius in the spherical approximation (14), which is here to be expanded through second order in $1 / r_{i j}$. Solving the equation $F_{1}=0$ yields

$$
\begin{aligned}
C_{1 i}= & -\left(\frac{\alpha_{i} \beta_{i}}{4 \sqrt{\alpha_{i} \beta_{i}}+\alpha_{i}+\beta_{i}}\right) \\
& \times \sum_{j \neq i} \frac{2 \sqrt{\alpha_{i} \beta_{i}}\left(\alpha_{j}+\beta_{j}\right)+\alpha_{i} \beta_{j}+\beta_{i} \alpha_{j}}{r_{i j}{ }^{2}} .
\end{aligned}
$$

Thus, the coefficients $C_{1 i}$ are second-order quantities. In [5], the coefficients $C_{n i}$ were determined numerically for uncharged black holes, and $\left|C_{n i}\right|$ was found to decrease as $n$ increases. Our results, 42 and (43), show analytically that here $\left|C_{n i}\right|$ decreases as $n$ increases due to its leading power in the inverse separations, $\left(1 / r_{i j}\right)^{2 n}$.

For the coefficients $C_{n i}$ in 42 and 43 , the horizon surface (40) approximates a prolate ellipsoid with one focus at $r=0$. This can be seen by linearizing the surface function $r_{\mathrm{e}}(\theta)$ of a prolate ellipsoid in the eccentricity $(\epsilon \ll 1)$,

$$
r_{\mathrm{e}}(\theta)=\frac{a\left(1-\epsilon^{2}\right)}{1+\epsilon \cos \theta} \simeq a-a \epsilon \cos \theta .
$$

The linearized form matches the horizon (40), so the horizon approximates an ellipsoid with semimajor axis $a=C_{0 i}=R_{i}$ and eccentricity $\epsilon=C_{1 i} / R_{i}$, as illustrated in Fig. 2 .

We now evaluate the horizon area $A_{i}$ in $(37)$ for the surface $r(\theta)$ in (40), using the metric function $f$ in (36). Expanding through second order in $1 / r_{i j}$, we find

$$
\begin{aligned}
\sqrt{\frac{A_{i}}{4 \pi}}= & m_{i}+\sqrt{\alpha_{i} \beta_{i}}\left(2+\sum_{j \neq i} \frac{\alpha_{j}+\beta_{j}}{r_{i j}}\right) \\
& -\frac{\sqrt{\alpha_{i} \beta_{i}}}{4}\left(\sum_{j \neq i} \frac{\alpha_{j}-\beta_{j}}{r_{i j}}\right)^{2},
\end{aligned}
$$

with $m_{i}$ given by (5) as a function of the other parameters $\left(\alpha_{i}, \beta_{i}, r_{i j}\right)$. The result (45) is the first-order area 24], plus second-order corrections.

Our next step is to obtain the energy $E\left(A_{i}, q_{i}, r_{i j}\right)$ as a function of the areas, charges, and separations. We evaluate the energy $E$ by a different method than in our first-order proof (Sec. III C), where we used the Newtonian form (26) of the energy, and expressed the masses $m_{i}$ in terms of the areas $A_{i}$. At second order, our setup is post-Newtonian, and we find it convenient to express the parameters $\alpha_{i}$ and $\beta_{i}$ in terms of the areas $A_{i}$ and charges $q_{i}$, and then evaluate the energy (7) in the form $E=\sum_{i}\left(\alpha_{i}+\beta_{i}\right)$.

Thus, we must solve the area equation (45) and the charge definition (6) for the parameters $\left(\alpha_{i}, \beta_{i}\right)$ as functions of the areas and charges $\left(A_{i}, q_{i}\right)$. For convenience 
in summarizing our results below, we define the following functions $\left(\mu_{i}, \mathcal{M}_{i}, \mathcal{Q}_{i}\right)$ of the areas and charges. We define the quantity $\mu_{i}$ as

$$
\mu_{i}=\sqrt{\frac{\pi}{A_{i}}}\left(\frac{A_{i}}{4 \pi}+q_{i}^{2}\right)
$$

which is our mass result 25 through first order. We define the two dimensionless quantities $\left(\mathcal{M}_{i}, \mathcal{Q}_{i}\right)$ as

$$
\mathcal{M}_{i}=\sum_{j \neq i} \frac{\mu_{j}}{r_{i j}}, \quad \mathcal{Q}_{i}=\sum_{j \neq i} \frac{q_{j}}{r_{i j}}
$$

We now solve for the parameters $\left(\alpha_{i}, \beta_{i}\right)$ using a perturbation approach: we write $\alpha_{i}$ and $\beta_{i}$ as their leadingorder values 9 , plus second-order corrections $\left(\widetilde{\alpha}_{i}, \widetilde{\beta}_{i}\right)$. We rephrase the leading-order values $(9)$ using the definitions 46) and (47). This gives

$$
\begin{aligned}
& \alpha_{i}=\frac{\left(\mu_{i}-q_{i}\right)}{2}\left[1-\frac{\left(\mathcal{M}_{i}+\mathcal{Q}_{i}\right)}{2}\right]+\widetilde{\alpha}_{i}, \\
& \beta_{i}=\frac{\left(\mu_{i}+q_{i}\right)}{2}\left[1-\frac{\left(\mathcal{M}_{i}-\mathcal{Q}_{i}\right)}{2}\right]+\widetilde{\beta}_{i} .
\end{aligned}
$$

It remains to solve for $\widetilde{\alpha}_{i}$ and $\widetilde{\beta}_{i}$ as functions of the areas and charges $\left(A_{i}, q_{i}\right)$. To this end, we insert (48) into the area result (45) and into the charge definition (6). After expanding through second order in $1 / r_{i j}$, we then find that 45 and (6) reduce to, respectively,

$$
K_{+} \widetilde{\alpha}_{i}+K_{-} \widetilde{\beta}_{i}=\varepsilon_{i}, \quad \widetilde{\beta}_{i}-\widetilde{\alpha}_{i}=\delta_{i} .
$$

We will summarize the coefficients $K_{ \pm}$and source terms $\left(\varepsilon_{i}, \delta_{i}\right)$ below. Solving the linear equations 49 yields the second-order corrections,

$$
\widetilde{\alpha}_{i}=\frac{\varepsilon_{i}-K_{-} \delta_{i}}{K_{+}+K_{-}}, \quad \widetilde{\beta}_{i}=\frac{\varepsilon_{i}+K_{+} \delta_{i}}{K_{+}+K_{-}} .
$$

The coefficients $K_{ \pm}$and source terms $\left(\varepsilon_{i}, \delta_{i}\right)$ are

$$
\begin{aligned}
K_{ \pm} & =1+\frac{\mu_{i} \pm q_{i}}{\sqrt{\mu_{i}^{2}-q_{i}^{2}}}, \\
\varepsilon_{i} & =\sqrt{\frac{A_{i}}{4 \pi}} \mathcal{S}_{i}-q_{i} \mathcal{T}_{i} \\
\delta_{i} & =q_{i} \mathcal{S}_{i}-\mu_{i} \mathcal{T}_{i} .
\end{aligned}
$$

For convenience, we have let $\mathcal{S}_{i}$ and $\mathcal{T}_{i}$ denote the following dimensionless second-order quantities,

$$
\begin{aligned}
& \mathcal{S}_{i}=\frac{\mathcal{M}_{i}{ }^{2}+\mathcal{Q}_{i}{ }^{2}}{4}+\sum_{j \neq i} \sum_{k \neq j} \frac{\left(\mu_{j} \mu_{k}-q_{j} q_{k}\right)}{4 r_{i j} r_{j k}}, \\
& \mathcal{T}_{i}=\frac{\mathcal{M}_{i} \mathcal{Q}_{i}}{2}-\sum_{j \neq i} \sum_{k \neq j} \frac{\left(\mu_{j} q_{k}-q_{j} \mu_{k}\right)}{4 r_{i j} r_{j k}} .
\end{aligned}
$$

Two simple results follow from using the second-order solutions for $\alpha_{i}$ and $\beta_{i}$. First, when we evaluate the mass $m_{i}$ in (5) using (48)-54, a significant number of terms cancel, and we find

$$
m_{i}=\mu_{i}
$$

with $\mu_{i}$ defined in 46 . Interestingly, this is the same as the mass function (25) through first order. Henceforth, we write $m_{i}$ in place of $\mu_{i}$. A second result is that, after using (50-54), the sum $\widetilde{\alpha}_{i}+\widetilde{\beta}_{i}$ reduces to

$$
\widetilde{\alpha}_{i}+\widetilde{\beta}_{i}=m_{i} \mathcal{S}_{i}-q_{i} \mathcal{T}_{i}
$$

We now evaluate the total energy $E=\sum_{i}\left(\alpha_{i}+\beta_{i}\right)$, as given by (7). Using (48) and (56) gives

$$
E=\sum_{i=1}^{N}\left[m_{i}-\frac{1}{2}\left(m_{i} \mathcal{M}_{i}-q_{i} \mathcal{Q}_{i}\right)+m_{i} \mathcal{S}_{i}-q_{i} \mathcal{T}_{i}\right] .
$$

The zeroth-order and first-order terms in (57) reproduce our previous result 26, which is the sum of the rest energies and the pairwise Newtonian and electrostatic potential energies. The second-order terms in (57), proportional to $\mathcal{S}_{i}$ and $\mathcal{T}_{i}$, are relativistic post-Newtonian corrections.

The energy (57) can be regarded as a function $E\left(A_{i}, q_{i}, r_{i j}\right)$ of the areas, charges, and separations. This follows from using the definitions of the quantities shown $\left(m_{i}, \mathcal{M}_{i}, \mathcal{Q}_{i}, \mathcal{S}_{i}, \mathcal{T}_{i}\right)$. Holding constant the charges $q_{i}$ and separations $r_{i j}$, we then find

$$
\frac{\partial E}{\partial A_{i}}=U_{i} \frac{\partial m_{i}}{\partial A_{i}}
$$

where

$$
\begin{aligned}
U_{i}= & -\mathcal{M}_{i}+\frac{1}{2}\left(\mathcal{M}_{i}^{2}+\mathcal{Q}_{i}^{2}\right) \\
& +\frac{3}{4} \sum_{j \neq i} \sum_{k \neq j} \frac{\left(m_{j} m_{k}-q_{j} q_{k}\right)}{r_{i j} r_{j k}} \\
& +\frac{1}{4} \sum_{j} \sum_{k \neq i, j} \frac{\left(m_{j} m_{k}-q_{j} q_{k}\right)}{r_{i k} r_{j k}} .
\end{aligned}
$$

In $(59)$, the leading term $\left(1-\mathcal{M}_{i}\right)$ is our first-order result 28) for $U_{i}$. The additional terms in (59), which are second order, are relativistic post-Newtonian contributions.

The remaining steps in our proof are now essentially the same as (29)-32 in our first-order proof (Sec. III C). We extremize the total energy $E$ in (57) by requiring $\partial E / \partial A_{i}=0$, which by (58) is equivalent to

$$
U_{i} \frac{\partial m_{i}}{\partial A_{i}}=0
$$

For sufficiently large separations, $U_{i} \neq 0$. In this case, the extremum 60 requires $\partial m_{i} / \partial A_{i}=0$. Evaluating this for 46 reduces to $\left|q_{i}\right|=\sqrt{A_{i} / 4 \pi}$. For this charge value, the mass 46 is $m_{i}=\sqrt{A_{i} / 4 \pi}=\left|q_{i}\right|$.

Thus, since the extremum 60 has produced the $N$ desired conditions, $\left|q_{i}\right|=m_{i}$, we have proved the extremum principle (2) through second order. As noted 
above, our proof requires $U_{i} \neq 0$. This condition is always satisfied at zeroth order (for which $U_{i}=1$ ), and it is satisfied through second order for sufficiently large separations $r_{i j}$ compared to the masses and charges, as seen by (59), and by (62) below. As in the first-order case, continuity of our perturbative approach with the zeroth-order limit $\left(U_{i}=1\right)$ requires $0<U_{i} \leq 1$.

We end this subsection by revisiting the interpretation of $U_{i}$. As in the first-order case (Sec. IIIC), note that $U_{i}$ can be obtained from the energy (57) in the form $E\left(m_{i}, q_{i}, r_{i j}\right)$, along with (58) and the chain rule,

$$
U_{i}=\frac{\partial E}{\partial m_{i}} .
$$

As in the first-order case, this motivates the interpretation of $U_{i}$ as the effective gravitational potential experienced by black hole $i$, including its rest energy. In this interpretation, it is not surprising that the gravitational potential (59) contains quadratic charge-dependent terms; this is because nonzero electric stress-energy (a quadratic function of the electric field) contributes to curving the geometry, and so contributes to the gravitational field. As an explicit example, for two black holes $(N=2)$,

$$
U_{1}=1-\frac{m_{2}}{r_{12}}+\frac{m_{2}^{2}+q_{2}^{2}+2\left(m_{1} m_{2}-q_{1} q_{2}\right)}{2 r_{12}{ }^{2}},
$$

and $U_{2}$ is similarly given by interchanging all subscripts $(1 \leftrightarrow 2)$. If we interpret $U_{1}$ as an effective gravitational potential, then the terms proportional to $m_{1} m_{2}-q_{1} q_{2}$ (which refer to black hole 1 itself) represent the nonlinear gravitational coupling in general relativity between black hole 1 and the other sources of energy $\left(m_{2}, q_{2}\right)$.

\section{E. Higher orders}

At higher orders, a proof of the extremum principle (2) can be expected to proceed similarly to Sec. IIID, so this subsection provides additional comments, rather than a full analysis. Through second order, as already noted in Sec. IIID the mass $m_{i}$ in 55 has the same form as the result (25) through first order: it is the same intrinsic function of the black hole's area $A_{i}$ and charge $q_{i}$, independent of the other black holes $(j \neq i)$. This result is perhaps unexpected, based on (5), where $m_{i}$ is a summation that involves the other black holes $(j \neq i)$. However, it could be anticipated physically, since the mass $m_{i}$ is specific to black hole $i$. Each mass $m_{i}$ contributes the the total energy,

$$
E=\sum_{i=1}^{N} m_{i}+E_{\text {int }} .
$$

Our goal is to evaluate and extremize the energy function $E\left(A_{i}, q_{i}, r_{i j}\right)$. From the observations above, the sum of the masses $m_{i}$ in (63) can be anticipated to take a simple form at higher orders. To evaluate $E$, it remains to consider the interaction energy, which we can view as the essential new task at each higher order, as a comparison of (26) and (57) illustrates; the steps will be similar to those following the evaluation of the area $A_{i}$ in Sec. IIID.

Through second order, our results of Sec. IIID show that that $E_{\text {int }}=0$ at the energy extremum, if all charges have the same sign. This is seen by letting let $q_{i}= \pm m_{i}$ for extremal charges, each with the same sign $( \pm)$. This gives $\mathcal{Q}_{i}= \pm \mathcal{M}_{i}$ and $\mathcal{T}_{i}= \pm \mathcal{S}_{i}$ from (47) and (54), respectively. Then several terms cancel in the energy (57), which reduces to $E=\sum_{i} m_{i}$, and shows that $E_{\text {int }}=0$.

It is promising to note that, at all orders, the following converse of the above result holds: if all charges are extremal and have the same sign, then the interaction energy vanishes, $E_{\mathrm{int}}=0$. This is most easily seen by substituting the extremal charge condition in the form (11) into the interaction energy (8). Since this is an exact result, it must hold at all orders, when all quantities are expanded in the inverse separations. The vanishing of $E_{\text {int }}$ for extremal same-sign charges is an exact supporting result, and is a precise statement about energy, like the extremum principle.

\section{ENERGY MINIMUM}

For sufficiently well separated black holes, as specified by the condition $U_{i}>0$ (see Secs. IIIC and IIID), it is straightforward to show that our energy extremum, 29p and (60), is a minimum. To show this, we verify that the second derivatives of $E$ are positive. Differentiating (27) and (58), holding constant the charges $q_{i}$ and separations $r_{i j}$, gives

$$
\frac{\partial^{2} E}{\partial A_{i}{ }^{2}}=U_{i} \frac{\partial^{2} m_{i}}{\partial A_{i}{ }^{2}}+\frac{\partial U_{i}}{\partial A_{i}} \frac{\partial m_{i}}{\partial A_{i}}
$$

We then evaluate $\partial^{2} m_{i} / \partial A_{i}{ }^{2}$ from (25) or (46), and we evaluate at the extremum $\left(\partial m_{i} / \partial A_{i}=0\right)$. This gives

$$
\frac{\partial^{2} E}{\partial A_{i}^{2}}=\frac{U_{i}}{8 \sqrt{\pi} A_{i}^{3 / 2}},
$$

where $U_{i}$ is to be evaluated with $m_{i}=\left|q_{i}\right|$. The condition $U_{i}>0$ ensures that 65 is positive, hence the energy extremum is a minimum.

If each charge $q_{i}$ has the same sign, it is also straightforward to verify that at the extremum, $E=|Q|$, where $Q$ is the total charge. This is seen as follows. Let $q_{i}= \pm m_{i}$ for extremal charges, each with the same sign $( \pm)$. Then

$$
Q=\sum_{i=1}^{N} q_{i}= \pm \sum_{i=1}^{N} m_{i} .
$$

As already noted in Sec. IIIE, for extremal same-sign charges $\left(q_{i}= \pm m_{i}\right)$, we also have

$$
E=\sum_{i=1}^{N} m_{i}= \pm Q=|Q| .
$$


The energy minimum and its extremum value are both consistent with the BPS bound in the energy inequality 9. that is satisfied by the Hartle-Hawking static solution [2]. In contrast to [9], we have minimized the energy $E\left(A_{i}, q_{i}, r_{i j}\right)$ as a function of physical variables, without using supersymmetry.

\section{CONCLUSION}

Our main result in this paper is an extremum principle, which derives the extremal charge condition $\left(\left|q_{i}\right|=m_{i}\right)$ for a set $N$ black holes (all initially at rest and arbitrarily charged) by extremizing the total energy $E\left(A_{i}, q_{i}, r_{i j}\right)$ with respect to the black hole horizon areas, at fixed charges and Euclidean separations. This principle is motivated by the first law of black hole mechanics, and is valid if the black holes are sufficiently well separated, as specified by the condition $U_{i}>0$, where $U_{i}$ can be interpreted as the effective gravitational potential experienced by black hole $i$.

Our energy extremum is taken at fixed Euclidean separations $r_{i j}$, rather than fixed proper distances between horizons. This is natural, in the sense that the proper distances are well known to become infinite for extremal charges, while the separations $r_{i j}$ remain finite.

If all of the black hole charges have the same sign, then our extremum principle is a type of variational principle, and augments a list of existing principles $[6]$, that interpret static black holes (here, the static Hartle-Hawking solution) as extrema of total energy. Our results are also consistent with the supersymmetric BPS energy minimum $(E=|Q|)$ of $[9$. Our derivation also shows how the corresponding substructure arises $\left(m_{i}=\left|q_{i}\right|\right)$ for each individual black hole. To our knowledge, our results provide the first energy interpretation of the static HartleHawking solution [2]. This interpretation requires neither the use of balanced forces, nor supersymmetry.

It would be interesting to consider the regime of very small black hole separations, which is beyond the scope of this paper. This would probably require numerical methods, and would involve an outermost common apparent horizon surrounding two nonextremal black holes that are sufficiently close to each other, similar to the case of uncharged black holes released at rest [5, 15]. The formation of a common apparent horizon has been studied numerically in the head-on collision of symmetric likecharged black holes [12] for charge-to-mass ratios in the range $0 \leq q / m \leq 0.98$.

In this paper, to locate a black hole's apparent horizon analytically, we have done so perturbatively, which is a well-known feature of the Brill-Lindquist geometry [5] that we have employed. One might wonder if a different geometry could be used instead; this appears to be unlikely. For example, in the conformally flat geometry found from Misner's well-known method of images [16, 17, each black hole's apparent horizon is designed to be an exact coordinate sphere in the flat background space, which is analytically convenient. This is achieved by constructing the solution as an infinite series (similar to the method of images in electrostatics). However, this approach does not permit black holes with extremal charge, since the infinite series solution must satisfy a convergence condition. This condition is rather formal in general; for two symmetric black holes with opposite charges, it reduces to the statement that the black holes are nonextremal [17. A similar restriction can be expected for same-sign charges. This suggests that the Brill-Lindquist geometry [5] is the unique family of nonextremal solutions that smoothly connects to the extremal Hartle-Hawking solution.

\section{ACKNOWLEDGMENTS}

This work was supported by the College of Science and Mathematics at California Polytechnic State University, San Luis Obispo. We thank Don Spector for useful discussions at the Kavli Institute for Theoretical Physics, University of California, Santa Barbara.
[1] G. A. Alekseev and V. A. Belinski, Phys. Rev. D76, 021501 (2007), arXiv:0706.1981

[2] J. B. Hartle and S. W. Hawking, Commun. Math. Phys. 26, 87 (1972).

[3] S. D. Majumdar, Phys. Rev. 72, 390 (1947)

[4] A. Papapetrou, Proc. Roy. Irish Acad. A 51, 191 (1947).

[5] D. R. Brill and R. W. Lindquist, Phys. Rev. 131, 471 (1963)

[6] S. W. Hawking, Commun. Math. Phys. 33, 323 (1973)

[7] D. Sudarsky and R. M. Wald, Phys. Rev. D46, 1453 (1992); P. T. Chrusciel and R. M. Wald, Commun. Math. Phys. 163, 561 (1994), arXiv:gr-qc/9304009.

[8] S. Fraser and D. M. Eardley, Phys. Rev. D92, 024031 (2015), arXiv:1408.4425

[9] G. W. Gibbons and C. M. Hull, Phys. Lett. B109, 190 (1982)
[10] L. Andrianopoli, R. D'Auria, S. Ferrara, and M. Trigiante, Lect. Notes Phys. 737, 661 (2008), arXiv:hepth/0611345

[11] S. W. Hawking, G. T. Horowitz, and S. F. Ross, Phys. Rev. D51, 4302 (1995), arXiv:gr-qc/9409013

[12] M. Zilhao, V. Cardoso, C. Herdeiro, L. Lehner, and U. Sperhake, Phys. Rev. D85, 124062 (2012), arXiv:1205.1063

[13] P. Ruback, Class. Quant. Grav. 5, L155 (1988)

[14] J. M. Bardeen, B. Carter, and S. Hawking, Commun. Math. Phys. 31, 161 (1973).

[15] N. T. Bishop, Gen. Rel. Grav. 14, 717 (1982)

[16] C. W. Misner, Ann. Phys. 24, 102 (1963).

[17] R. W. Lindquist, J. Math. Phys. 4, 938 (1963) 